\title{
Dynamics Analysis of a Betel Nut Addiction Spreading Model on Scale-Free Networks
}

\author{
He Wang, ${ }^{1}$ Tao Li $\mathbb{D}^{1,2}{ }^{1,2}$ Xinming Cheng, ${ }^{3}$ Yu Kong, ${ }^{1}$ and Yangmei Lei ${ }^{1}$ \\ ${ }^{1}$ School of Electronics and Information, Yangtze University, Jingzhou 434023, China \\ ${ }^{2}$ National Demonstration Center for Experimental Electrical and Electronic Education, Yangtze University, \\ Jingzhou 434023, China \\ ${ }^{3}$ School of Automation, Central South University, Changsha 410083, China \\ Correspondence should be addressed to Tao Li; taohust2008@163.com
}

Received 17 July 2020; Revised 9 September 2020; Accepted 16 September 2020; Published 27 September 2020

Academic Editor: Frédéric Magoulès

Copyright (C) $2020 \mathrm{He}$ Wang et al. This is an open access article distributed under the Creative Commons Attribution License, which permits unrestricted use, distribution, and reproduction in any medium, provided the original work is properly cited.

\begin{abstract}
Medical research has shown that overeating betel nut can be addictive and can damage health. More serious cases may cause mouth cancer and other diseases. Even worse, people's behavior habit of chewing betel nut may influence each other through social interaction with direct or indirect ways, such as face-to-face communication, Facebook, Twitter, microblog, and WeChat, which leads to the spreading phenomenon of betel nut addiction. In order to investigate the dynamic spreading characteristics of betel nut addiction, a PMSR (Potential-Mild-Severe-Recovered) betel nut addiction spreading model is presented on scale-free networks. The basic reproductive number $R_{0}$ and equilibria are derived. Theoretical results indicate that the basic reproductive number $R_{0}$ is significantly dependent on the topology of the underlying networks, and some influence parameters. The existence of equilibria is determined by the basic reproductive number $R_{0}$. Furthermore, we prove that if $R_{0}<1$ the addiction-elimination equilibrium is globally asymptotically stable. If $R_{0}>1$, the betel nut addiction spreading is permanent, and the addictionprevailing equilibrium is globally attractive. Finally, numerical simulations confirm the theoretical analysis results.
\end{abstract}

\section{Introduction}

The habit of chewing betel nut is common in many places around the world [1-8]. It is worth noting that overeating betel nut can be addictive and is harmful to health $[8,9]$. It is found that betel nut affects the health of the nervous, gastrointestinal, metabolic, respiratory, and reproductive systems. More serious cases may cause mouth cancer and other diseases. Betel nut is classified in the first category of carcinogens by the International Agency for Research on Cancer [9-11]. Even worse, people's behavior habit of chewing betel nut may influence each other through social contact, which leads to the spreading phenomenon of betel nut addiction. The widespread spread of betel nut addiction, in turn, exacerbates the damage to people's health and even the whole society.

It is very important to control the spreading phenomenon of betel nut addiction. In recent years, the research on betel nut addiction has attracted the attention of many scholars and researchers. Murphy et al. confirmed that the formation of the "chewing betel nut" habit could be due to exposure to and influence by betel nut chewers through statistical analysis [12]. As indicated in [13], the cultural differences can affect the spreading of betel nut addiction in society. Ghani et al. identified the factors which influence the development of betel nut addiction. They also proposed the health policies to prevent addiction [14]. Moss et al. [15] showed that the critical factors of addiction were contact with addicts and the self-prevention consciousness. Many researchers studied the addictiveness and harmfulness of the "chewing betel nut" habit [16-19], but few focused on the spreading dynamics of betel nut addiction. There are some studies in social information and disease spreading dynamics [20-25]. Liu et al. studied the spreading dynamics of cyber violence [26]. Barabási and Albert studied the impact of neighboring infection on the computer virus spread [27]. 
These studies can give some help for the analysis of the spreading dynamics of betel nut addiction. Through the study of addiction spreading dynamics, we can comprehensively and systematically learn about the addiction spreading mechanism and influence factors, which is helpful to control the spread of betel nut addiction. Meanwhile, some researchers found that the scale-free property is an important property of social networks [28-32]. Obviously, the spread of betel nut addiction is based on social networks. So, based on scale-free networks, we study the spreading dynamics of betel nut addiction in the paper. Apparently, the marketing strategy of betel nut has a great influence on sales, and at the same time, it affects the spreading of betel nut addiction. Taking into account the influence of betel nut advertising campaigns and the heterogeneity of underlying spreading networks, we present a new comprehensively PMSR (Potential-Mild-Severe-Recovered) betel nut addiction spreading model.

The rest of the paper is as follows. In Section 2, the PMSR betel nut addiction spreading model is proposed and described. In Section 3, the basic reproductive number and equilibriums are derived at first. Then, we analyze the globally asymptotic stability of addiction-elimination equilibrium, the permanence of the addiction spreading, and the global attractivity of addiction-prevailing equilibrium. Section 4 presents the results of our numerical simulation. Finally, we conclude the paper in Section 5.

\section{Model Formulation}

We present a new comprehensively PMSR (Potential-MildSevere-Recovered) betel nut addiction spreading model. The model has the spread sketch in Figure 1. In the model, nodes are used to stand for individuals, and edges are used to stand for the relationships between individuals. The whole population is divided into four distinct classes, namely, potential individuals $(P)$, mild addicts $(M)$, severe addicts $(S)$, and recovered individuals $(R)$. $P$ nodes refer to the people who do not have addictive behavior of chewing betel nut; $M$ nodes refer to the people who have mild addiction of the "chewing betel nut" habit; $S$ nodes refer to the people who have severe addiction of the "chewing betel nut" habit; and $R$ nodes refer to the people who get rid of the addiction.

In the spreading process of betel nut addiction, these states are subjected to the following rules:

(1) If a potential individual is influenced by a mild addict with direct or indirect ways, such as face-to-face communication, Facebook, Twitter, microblog, and WeChat, then he or she will convert into a mild addict with probability $\rho_{1}$. Similarly, if a potential individual is influenced by a severe addict with direct or indirect ways, then he or she will convert into a mild addict with probability $\rho_{2}$.

(2) The parameter $\alpha$ is defined as an influence parameter of betel nut advertisements. In real life, the marketing strategy of betel nut will influence people to try to purchase betel nut.

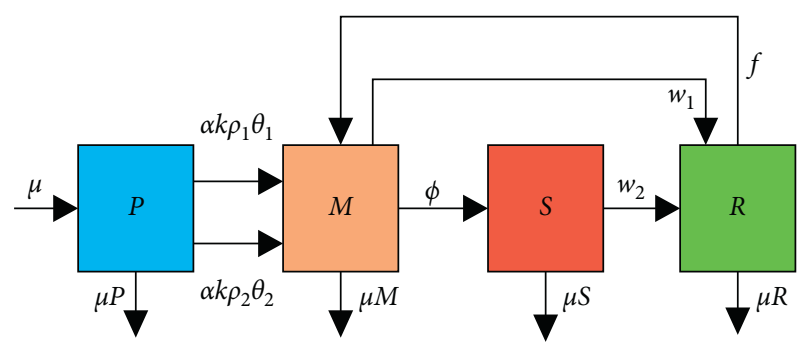

FIgure 1: The flow diagram of the PMSR model.

(3) A mild addict may convert into a severe addict with probability $\varphi$ or get rid of the addiction with probability $w_{1}$.

(4) The severe addict may get rid of the addiction with probability $w_{2}$.

(5) Considering the relapse of addiction, a recovered individual may convert into a mild addict with probability $f$.

(6) Here, we assume that the immigration rate equals the emigration rate, and the rate constant is $\mu$.

We define $P_{k}(t), M_{k}(t), S_{k}(t)$, and $R_{k}(t)$ as the relative densities of potential individuals, mild addicts, severe addicts, and recovered individuals nodes of degree $k$ at time $t$, respectively. According to the above description and assumption, we can get the PMSR model as follows:

$$
\left\{\begin{array}{l}
\frac{\mathrm{d} P_{k}(t)}{\mathrm{d} t}=\mu-k \alpha\left(\rho_{1} \theta_{1}+\rho_{2} \theta_{2}\right) P_{k}(t)-\mu P_{k}(t), \\
\frac{\mathrm{d} M_{k}(t)}{\mathrm{d} t}=k \alpha\left(\rho_{1} \theta_{1}+\rho_{2} \theta_{2}\right) P_{k}(t)+f R_{k}(t) \\
-\left(\varphi+\mu+w_{1}\right) M_{k}(t), \\
\frac{\mathrm{d} S_{k}(t)}{\mathrm{d} t}=\varphi M_{k}(t)-\left(w_{2}+\mu\right) S_{k}(t), \\
\frac{\mathrm{d} R_{k}(t)}{\mathrm{d} t}=\omega_{2} S_{k}(t)+w_{1} M_{k}(t)-(f+\mu) R_{k}(t),
\end{array}\right.
$$

where $\theta_{1}(t)$ and $\theta_{2}(t)$ denote the probability of a contact pointing to a mild addict and a severe addict, respectively, and

$$
\begin{aligned}
& \theta_{1}(t)=\frac{1}{\langle k\rangle} \sum_{k=1}^{\infty} k H(k) M_{k}(t), \\
& \theta_{2}(t)=\frac{1}{\langle k\rangle} \sum_{k=1}^{\infty} k H(k) S_{k}(t) .
\end{aligned}
$$

Here, $\langle k\rangle=\sum_{k} k H(k)$ represents the average degree values of the network and $H(k)$ represents the degree distribution. $M(t)=\sum_{k} H(k) M_{k}(t)$ is the density of all the mild addicts and $S(t)=\sum_{k} H(k) S_{k}(t)$ is the density of all the severe addicts. 
According to the normalization conditions, we have

$$
P_{k}(t)+M_{k}(t)+S_{k}(t)+R_{k}(t)=1 .
$$

Obviously, the initial values for system (1) are as follows:

$$
\begin{aligned}
P_{k}(0) & >0, \\
M_{k}(0) & \geq 0, \\
S_{k}(0) & \geq 0, \\
C_{k}(0) & \geq 0, \quad k=1,2, \ldots, n .
\end{aligned}
$$

Then, we obtain that

$$
\begin{aligned}
P_{k}(t) & >0, \\
M_{k}(t) & \geq 0, \\
S_{k}(t) & \geq 0, \\
C_{k}(t) & \geq 0, \quad k=1,2, \ldots, n \text { and } t \geq 0 .
\end{aligned}
$$

\section{Stability Analysis of the Model}

In this section, we analyze the dynamic properties of the PMSR betel nut addiction spreading model.

Theorem 1. According to system (1), the basic reproductive number is defined as follows:

$$
R_{0}=\frac{\alpha(f+\mu)\left[\rho_{1}\left(\mu+w_{2}\right)+\rho_{2} \varphi\right]}{\left[\left(w_{2}+\mu\right)\left(w_{1}+f+\mu\right)+\varphi\left(w_{2}+f+\mu\right)\right] \mu} .
$$

There always exists an addiction-elimination equilibrium $E_{0}(1,0,0,0)$, and if $R_{0}>1$, system (1) has a unique addictionprevailing equilibrium $E_{+}\left(P_{k}^{*}, M_{k}^{*}, S_{k}^{*}, R_{k}^{*}\right)$.

Proof. It can be easy to find that system (1) satisfies $R_{k}(t)=1-P_{k}(t)-M_{k}(t)-S_{k}(t)$. According to system (1), we can get

$$
\left\{\begin{array}{l}
\frac{\mathrm{d} P_{k}(t)}{\mathrm{d} t}=\mu-k \alpha\left(\rho_{1} \theta_{1}+\rho_{2} \theta_{2}\right) P_{k}(t)-\mu P_{k}(t), \\
\frac{\mathrm{d} M_{k}(t)}{\mathrm{d} t}=k \alpha\left(\rho_{1} \theta_{1}+\rho_{2} \theta_{2}\right) P_{k}(t) \\
\quad+f\left(1-P_{k}(t)-M_{k}(t)-S_{k}(t)\right)-\left(\varphi+\mu+w_{1}\right) M_{k}(t), \\
\frac{\mathrm{d} S_{k}(t)}{\mathrm{d} t}=\varphi M_{k}(t)-(d+\mu) S_{k}(t) .
\end{array}\right.
$$

Obviously, there is an addiction-elimination equilibrium $E_{0}(1,0,0,0)$ in system (1). It is easy to verify that system (8) satisfies the conditions $\left(A_{1}\right)-\left(A_{5}\right)$ in [33]. By using the next generation matrix method [33], system (8) can get

$$
\frac{\mathrm{d} x}{\mathrm{~d} t}=Z(x)-V(x)
$$

where

$$
\begin{aligned}
x & =\left(P_{k}, M_{k}, S_{k}\right)^{T}, \\
Z(x) & =\left(\begin{array}{c}
-k \alpha\left(\rho_{1} \theta_{1}(t)+\rho_{2} \theta_{2}(t)\right) P_{k}(t) \\
k \alpha\left(\rho_{1} \theta_{1}(t)+\rho_{2} \theta_{2}(t)\right) P_{k}(t) \\
0
\end{array}\right), \\
V(x) & =\left(\begin{array}{c}
\left(w_{1}+\mu+\varphi\right) P_{k}(t)-f C_{k}(t) \\
\left(w_{2}+\mu\right) S_{k}(t)-\varphi M_{k}(t)
\end{array}\right) .
\end{aligned}
$$

At the addiction-elimination equilibrium $E_{0}$, the Jacobian matrices of $Z(x)$ and $V(x)$ can get

$$
\begin{aligned}
Z & =J Z\left(E_{0}\right) \\
& =\left(\begin{array}{ccc}
0 & Z_{12} & Z_{13} \\
0 & Z_{22} & Z_{23} \\
0 & 0 & 0
\end{array}\right), \\
V & =J V\left(E_{0}\right) \\
& =\left(\begin{array}{ccc}
V_{11} & 0 & 0 \\
V_{21} & V_{22} & V_{23} \\
0 & V_{32} & V_{33}
\end{array}\right),
\end{aligned}
$$

where

$$
Z_{12}=\frac{-\alpha \rho_{1}}{\langle k\rangle}\left[\begin{array}{c}
1 \\
2 \\
\vdots \\
n
\end{array}\right]\left[\begin{array}{llll}
1 P(1) & 2 P(2) & \cdots & n P(n)
\end{array}\right],
$$$$
Z_{13}=\frac{-\alpha \rho_{2}}{\langle k\rangle}\left[\begin{array}{c}
1 \\
2 \\
\vdots \\
n
\end{array}\right]\left[\begin{array}{llll}
1 P(1) & 2 P(2) & \cdots & n P(n)
\end{array}\right] \text {, }
$$$$
Z_{22}=\frac{\alpha \rho_{1}}{\langle k\rangle}\left[\begin{array}{c}
1 \\
2 \\
\vdots \\
n
\end{array}\right]\left[\begin{array}{llll}
1 P(1) & 2 P(2) & \cdots & n P(n)
\end{array}\right],
$$

$$
Z_{23}=\frac{\alpha \rho_{2}}{\langle k\rangle}\left[\begin{array}{c}
1 \\
2 \\
\vdots \\
n
\end{array}\right]\left[\begin{array}{llll}
1 P(1) & 2 P(2) & \cdots & n P(n)
\end{array}\right] .
$$


Here,

$$
\begin{aligned}
& V_{11}=\mu I, \\
& V_{21}=f I, \\
& V_{22}=\left(w_{1}+\mu+\varphi+f\right) I, \\
& V_{23}=f I, \\
& V_{32}=-\varphi I, \\
& V_{33}=\left(w_{2}+\mu\right) I,
\end{aligned}
$$

where $I$ is the identity matrix. So, we can get the basic reproductive number:

$$
\begin{aligned}
R_{0} & =\rho\left(Z V^{-1}\right) \\
& =\frac{\left\langle k^{2}\right\rangle}{\langle k\rangle} \frac{\alpha(f+\mu)\left[\rho_{1}\left(\mu+w_{2}\right)+\rho_{2} \varphi\right]}{\left[\left(w_{2}+\mu\right)\left(w_{1}+f+\mu\right)+\varphi\left(w_{2}+f+\mu\right)\right] \mu},
\end{aligned}
$$

where $\left\langle k^{2}\right\rangle=\sum_{k} k^{2} P(k)$.

Next, it is clear that system (1) has an equilibrium $E_{0}=\{(1,0,0,0)\}_{k}$. To get the addiction-prevailing equilibrium $E^{*}\left(P_{k}^{*}, M_{k}^{*}, S_{k}^{*}, R_{k}^{*}\right)$, system (1) satisfies

$$
\begin{aligned}
\frac{\mathrm{d} P_{k}(t)}{\mathrm{d} t} & =0, \\
\frac{\mathrm{d} M_{k}(t)}{\mathrm{d} t} & =0, \\
\frac{\mathrm{d} S_{k}(t)}{\mathrm{d} t} & =0, \\
\frac{\mathrm{d} R_{k}(t)}{\mathrm{d} t} & =0 .
\end{aligned}
$$

So, we can know

$$
\left\{\begin{array}{l}
\mu-k \alpha\left(\rho_{1} \theta_{1}+\rho_{2} \theta_{2}\right) P_{k}(t)-\mu P_{k}(t)=0, \\
k \alpha\left(\rho_{1} \theta_{1}+\rho_{2} \theta_{2}\right) P_{k}(t)+f R_{k}(t)-\left(\varphi+\mu+w_{1}\right) M_{k}(t)=0, \\
\varphi M_{k}(t)-\left(w_{2}+\mu\right) S_{k}(t)=0, \\
w_{2} S_{k}(t)+w_{1} M_{k}(t)-(f+\mu) R_{k}(t)=0 .
\end{array}\right.
$$

According to the above equations, we get

$$
\left\{\begin{array}{l}
P_{k}=\frac{\mu\left[w_{1}\left(w_{2}+\mu\right)+(f+\mu)\left(w_{2}+\mu\right)+\varphi\left(w_{2}+f+\mu\right)\right]}{k \alpha\left(\rho_{1} \theta_{1}+\rho_{2} \theta_{2}\right)\left(w_{2}+\mu\right)(f+\mu)} M_{k}, \\
S_{k}=\frac{\varphi}{w_{2}+\mu} M_{k}, \\
R_{k}=\frac{\left(w_{2}+\mu\right) w_{1}+\varphi w_{2}}{\left(w_{2}+\mu\right)(f+\mu)} M_{k} .
\end{array}\right.
$$

By using the normalization condition $P_{k}^{*}(t)+M_{k}^{*}(t)+S_{k}^{*}(t)+R_{k}^{*}(t)=1$, we get

$$
\left\{\begin{array}{l}
P_{k}^{*}=\frac{\mu\left[w_{1}\left(w_{2}+\mu\right)+(f+\mu)\left(w_{2}+\mu\right)+\varphi\left(w_{2}+f+\mu\right)\right]}{k a\left(\rho_{1} \theta_{1}^{*}+\rho_{2} \theta_{2}^{*}\right)\left(w_{2}+\mu\right)(f+\mu)} M_{k}^{*}, \\
M_{k}^{*}=\frac{(f+\mu) w_{1}\left(w_{2}+\mu\right)+\mu\left[(f+\mu)\left(w_{2}+\mu\right)+\varphi\left(w_{2}+f+\mu\right)\right]}{\left(w_{2}+\mu\right)(f+\mu)\left(w_{1}+\mu+\varphi\right)} \\
S_{k}^{*}=\frac{\varphi}{w_{2}+\mu} M_{k}^{*}, \\
R_{k}^{*}=\frac{\left(w_{2}+\mu\right) w_{1}+\varphi w_{2}}{\left(w_{2}+\mu\right)(f+\mu)} M_{k}^{*} .
\end{array}\right.
$$

And, for $\theta_{1}^{*}=1 /\langle k\rangle \sum_{k=1}^{\infty} k P(k) M_{k}^{*}(t), \quad \theta_{2}^{*}=1 /\langle k\rangle$ $\sum_{k=1}^{\infty} k P(k) S_{k}^{*}(t)$, we get

$$
\begin{aligned}
& \theta_{1}^{*}=\frac{w_{2}+\mu}{\varphi} \theta_{2}^{*}, \\
& \theta_{2}^{*}=\frac{\varphi}{w_{2}+\mu} \theta_{1}^{*} .
\end{aligned}
$$

By substituting the second equation of system (16) into equation (2), we get

$$
\begin{aligned}
\theta_{1}^{*} & =\frac{1}{\langle k\rangle} \sum_{k=1}^{\infty} k P(k) M_{k}^{*}(t) \\
& =\frac{1}{\langle k\rangle} \sum_{k=1}^{\infty} k P(k) \frac{k \alpha\left(\rho_{1} \theta_{1}^{*}+\rho_{2} \theta_{2}^{*}\right)\left(w_{2}+\mu\right)(f+\mu)}{\left[k \alpha\left(\rho_{1} \theta_{1}^{*}+\rho_{2} \theta_{2}^{*}+\mu\right)\right]\left[\left(w_{2}+\mu\right)(f+\mu)+\varphi(f+\mu)\right]} \\
& =\frac{1}{\langle k\rangle} \sum_{k=1}^{\infty} k^{2} P(k) \frac{k \alpha(f+\mu)\left(w_{2}+\mu\right)\left[\rho_{1}\left(w_{2}+\mu\right)+\rho_{2} \varphi\right] \theta_{1}^{*}}{k \alpha\left(w_{2}+\mu\right)(f+\mu)\left[\rho_{1}\left(w_{2}+\mu\right)+\rho_{2} \varphi\right] \theta_{1}^{*}+A_{k}} \\
& =f\left(\theta_{1}^{*}\right),
\end{aligned}
$$


where

$$
\begin{aligned}
A_{k}= & \mu\left(w_{2}+\mu\right)(f+\mu)\left[\left(w_{2}+\mu\right)+\varphi+k \alpha \varphi\right] \\
& +k \alpha \varphi(f+\mu)\left[\left(w_{2}+\mu\right) \rho_{1}+\varphi \rho_{2}\right] \theta_{1}^{*} .
\end{aligned}
$$

Obviously, $\theta_{1}^{*}=0$ satisfies system (20). System (20) has a unique nontrivial solution provided that

$$
\left.\frac{\mathrm{d} f\left(\theta_{1}^{*}\right)}{\mathrm{d} \theta_{1}^{*}}\right|_{\Theta_{1}^{*}=0}>1, \quad f(1) \leq 1 .
$$

So, we get

$$
R_{0}=\frac{\left\langle k^{2}\right\rangle}{\langle k\rangle} \frac{\alpha(f+\mu)\left[\rho_{1}\left(\mu+w_{2}\right)+\rho_{2} \varphi\right]}{\left[\left(w_{2}+\mu\right)\left(w_{1}+f+\mu\right)+\varphi\left(w_{2}+f+\mu\right)\right] \mu}>1 .
$$

Thus, a unique nontrivial solution exists if and only if $R_{0}>1$ According to equation (17), we know

$$
\begin{aligned}
& 0<P_{k}^{\infty}<1, \\
& 0<M_{k}^{\infty}<1, \\
& 0<S_{k}^{\infty}<1, \\
& 0<R_{k}^{\infty}<1 .
\end{aligned}
$$

Therefore, the addiction-prevailing equilibrium $E^{*}\left(P_{k}^{*}, M_{k}^{*}, S_{k}^{*}, R_{k}^{*}\right)$ is well defined. Then, when $R_{0}>1$, there is a unique positive equilibrium $E^{*}\left(P_{k}^{*}, M_{k}^{*}, S_{k}^{*}, R_{k}^{*}\right)$. The proof is completed.

Remark 1 . The basic reproductive number $R_{0}$ is obtained by equation (23), which depends on the fluctuations of the degree distribution $\left\langle k^{2}\right\rangle /\langle k\rangle$ and some model parameters. When the heterogeneity of the degree distribution $\left\langle k^{2}\right\rangle /\langle k\rangle$ is larger, the basic reproductive number $R_{0}$ is greater, i.e., the larger heterogeneity of the degree distribution can promote the spreading of betel nut addiction. Obviously, as the influence parameter $\alpha$ of betel nut advertisements increases, the basic reproductive number $R_{0}$ increases.

Theorem 2. If $R_{0}<1$, the addiction-elimination equilibrium $E_{0}$ is globally asymptotically stable. If $R_{0}>1$, the addiction spreading phenomenon is persistent, i.e., there exists a constant $\eta>0$, such that

$$
\begin{aligned}
\lim _{t \longrightarrow \infty} \inf (M(t)+S(t))= & \lim _{t \longrightarrow \infty} \inf \sum_{k} H(k)\left(M_{k}(t)\right. \\
& \left.+S_{k}(t)\right) \geq \eta .
\end{aligned}
$$

Proof. For the addiction-elimination equilibrium, system (8) has the Jacobian matrix as follows:

$$
J=\left(\begin{array}{ccc}
A_{11} & \cdots & A_{1 n} \\
\vdots & \ddots & \vdots \\
A_{n 1} & \cdots & A_{n n}
\end{array}\right),
$$

where

$$
\begin{aligned}
& A_{11}=\left(\begin{array}{ccc}
\alpha \rho_{1} P_{1}-\left(w_{1}+\mu+\varphi\right) & \alpha \rho_{2} P_{1} & f \\
\varphi & -\left(w_{2}+\mu\right) & 0 \\
w_{1} & w_{2} & -(f+\mu)
\end{array}\right) \text {, } \\
& A_{1 n}=\left(\begin{array}{ccc}
\alpha \rho_{1} P_{n} & \alpha \rho_{2} P_{n} & 0 \\
0 & 0 & 0 \\
0 & 0 & 0
\end{array}\right) \text {, } \\
& A_{n 1}=\left(\begin{array}{ccc}
n \alpha \rho_{1} P_{n} & n \alpha \rho_{2} P_{n} & 0 \\
0 & 0 & 0 \\
0 & 0 & 0
\end{array}\right) \text {, } \\
& A_{n n}=\left(\begin{array}{ccc}
n \alpha \rho_{1} P_{n}-\left(w_{1}+\mu+\varphi\right) & n \alpha \rho_{2} P_{n} & f \\
\varphi & -\left(w_{2}+\mu\right) & 0 \\
w_{1} & w_{2} & -(f+\mu)
\end{array}\right) \text {. }
\end{aligned}
$$

So, the characteristic polynomial of the addictionelimination equilibrium is

$$
\begin{aligned}
& \left(\lambda+w_{1}+\mu+\varphi\right)^{n-1}\left(\lambda+w_{2}+\mu\right)^{n-1}\left(\lambda^{2}+p \lambda+q\right) \\
& \cdot(\lambda+f+\mu)^{n-1}=0,
\end{aligned}
$$

where

$$
\begin{aligned}
p= & \left(w_{2}+\mu\right)\left(w_{1}+f+\mu\right)+\varphi\left(w_{2}+f+\mu\right) \\
& +(f+\mu)\left(w_{1}+\mu\right)+\mu\left(w_{2}+\mu+\varphi\right) \\
& -\left[\rho_{1}\left(f+\mu+w_{2}+\mu\right)+\rho_{2} \varphi \alpha\right] \sum_{i=1}^{n} i P_{i}-f w_{1}, \\
q= & \left(w_{1}+\mu+\varphi\right)\left(w_{2}+\mu\right)(f+\mu)+f \varphi w_{2} \\
& -\alpha(f+\mu)\left[\rho_{1}\left(w_{2}+\mu\right)+\rho_{2} \varphi\right] \sum_{i=1}^{n} i P_{i}-f w_{1}\left(w_{2}+\mu\right) .
\end{aligned}
$$

When $R_{0}<1$, we can get

$R_{0}=\frac{\left\langle k^{2}\right\rangle}{\langle k\rangle} \frac{\alpha(f+\mu)\left[\rho_{1}\left(\mu+w_{2}\right)+\rho_{2} \varphi\right]}{\left[\left(w_{2}+\mu\right)\left(w_{1}+f+\mu\right)+\varphi\left(w_{2}+f+\mu\right)\right] \mu}<1$.

It also means

$$
\begin{gathered}
{\left[\left(w_{2}+\mu\right)\left(w_{1}+f+\mu\right)+\varphi\left(w_{2}+f+\mu\right)\right] \mu} \\
>\alpha(f+\mu)\left[\rho_{1}\left(\mu+w_{2}\right)+\rho_{2} \varphi\right] \sum_{i=1}^{n} i P_{i} .
\end{gathered}
$$

In other words, we get $p>0$ and $q>0$. According to the above proof, the real eigenvalues $\lambda$ of matrix $J$ are all negative when $R_{0}<1$. Furthermore, if and only if $R_{0}>1$, there is a unique positive eigen value $\lambda$ of matrix $J$. By using the Perron-Frobenius theorem, the maximal real part of all eigenvalues of $\lambda$ is positive only if $R_{0}>1$. Through the theorem of Lajmanovich and York [34], we can get the results. The proof is completed. 
Lemma 1 (see [35]). If $a>0, b>0$ and $d x(t) / d t \geq b-a x$, when $t \geq 0$ and $x(0) \geq 0$, we have $\lim _{t \rightarrow \infty} \inf x(t) \geq b / a$; if $a>0, b>0$ and $d x(t) / d t \leq b-a x$, when $t \geq 0$ and $x(0) \geq 0$, we have $\lim _{t \rightarrow \infty} \sup x(t) \leq b / a$.

Next, the global attractivity of the addiction-prevailing equilibrium is discussed. The main result is given in the following theorem.

Theorem 3. Suppose that $\left(P_{k}(t), M_{k}(t), S_{k}(t)\right)$ is a solution of system (8) satisfying initial conditions $M_{k}(t)>0$ or $S_{k}(t)>0$. If $R_{0}>1$, then

$$
\lim _{t \longrightarrow \infty}\left(P_{k}(t), M_{k}(t), S_{k}(t)\right)=\left(P_{k}^{*}(t), M_{k}^{*}(t), S_{k}^{*}(t)\right),
$$

where $\left(P_{k}^{*}(t), M_{k}^{*}(t), S_{k}^{*}(t)\right)$ is the addiction-prevailing equilibrium of system (8) for $k=1,2, \ldots, n$.

Proof. In the course of the proving, we followed the methods of reference [35]. In the following, we suppose that $k$ is an integer between 1 and $n$. According to Theorem 2, there exists a positive constant $0<\tau<1 / 3$ and a large enough constant $T>0$ such that $M_{k}(t) \geq \tau$ and $S_{k}(t) \geq \tau$ for $t>T$. Therefore, $\theta_{1}(t)>\tau, \theta_{2}(t)>\tau$ for $t>T$. Substituting this into the first equation of system (8), it is easy to get

$$
\begin{aligned}
\frac{\mathrm{d} P_{k}(t)}{\mathrm{d} t} & =\mu-k \alpha\left(\rho_{1} \theta_{1}+\rho_{2} \theta_{2}\right) P_{k}(t)-\mu P_{k}(t) \\
& \leq \mu-\left[k \alpha\left(\rho_{1}+\rho_{2}\right) \tau+\mu\right] P_{k}(t), \quad t>T .
\end{aligned}
$$

From Lemma 1, according to the standard comparison theorem of differential equation theory, for any given positive constant $0<\tau_{1}<k\left(\rho_{1}+\rho_{2}\right) \tau /\left(\mu+k a\left(\rho_{1}+\rho_{2}\right) \tau\right)$, there exists $t_{1}>T$, such that $P_{k}(t) \leq X_{k}^{(1)}-\tau_{1}$ for $t>t_{1}$, where

$$
X_{k}^{(1)}=\frac{\mu}{\mu+k \alpha\left(\rho_{1}+\rho_{2}\right) \tau}+2 \tau_{1}<1 .
$$

From system (8), it is easy to obtain

$$
\begin{aligned}
\frac{\mathrm{d} M_{k}}{\mathrm{~d} t}= & {\left[k \alpha\left(\rho_{1} \theta_{1}+\rho_{2} \theta_{2}\right)+f\right]\left(1-M_{k}(t)\right) } \\
& -\left(w_{1}+\mu+\varphi\right) M_{k}(t), \quad t>t_{1} .
\end{aligned}
$$

So, the constant $0<\tau_{2}<\min \left\{1 / 2, w_{1}+\mu+\right.$ $\left.\varphi / 2\left[k \alpha\left(\rho_{1}+\rho_{2}\right) \tau+w_{1}+\mu+\varphi+f\right]\right\}$, there exists $t_{2}>t_{1}$, so $M_{k}(t) \leq Y_{k}^{(1)}-\tau_{2}$ for $t>t_{2}$, where

$$
Y_{k}^{(1)}=\frac{2 k\left(\rho_{1}+\rho_{2}\right) \tau+f}{2 k\left(\rho_{1}+\rho_{2}\right) \tau+f+w_{1}+\mu+\varphi}+2 \tau_{2}<1 .
$$

From system (8), it is easy to obtain

$$
\frac{\mathrm{d} S_{k}(t)}{\mathrm{d} t} \leq \varphi\left(1-S_{k}(t)\right)-\left(w_{2}+\mu\right) S_{k}(t), \quad t>t_{2} .
$$

Thus, for constant $0<\tau_{3}<\min \left\{1 / 3, \tau_{2}, w_{2}+\right.$ $\left.\mu / 2\left(\varphi+w_{2}+\mu\right)\right\}$, there exists $t_{3}>t_{2}$, so $S_{k}(t) \leq Z_{k}^{(1)}-\tau_{3}$ for $t>t_{3}$, where

$$
Z_{k}^{(1)}=\frac{\varphi}{\varphi+w_{2}+\mu}+2 \tau_{3}<1 .
$$

Then, replacing $M_{k}(t) \leq Y_{k}^{(1)}-\tau_{2}$ and $S_{k}(t) \leq Z_{k}^{(1)}-\tau_{3}$ into the first equation of system (8), we get

$$
\begin{aligned}
\frac{\mathrm{d} P_{k}(t)}{\mathrm{d} t} & =\mu-k \alpha\left(\rho_{1} \theta_{1}(t)+\rho_{2} \theta_{2}(t)\right) P_{k}(t)-\mu P_{k}(t) \\
& \geq \mu-\left[k \alpha\left(\rho_{1}+\rho_{2}\right) \tau+\mu\right] P_{k}(t), \quad t>T .
\end{aligned}
$$

Thus, for constant $0<\tau_{4}<\min \left\{1 / 4, \mu / 2\left[k \alpha\left(\rho_{1}+\rho_{2}\right) \tau+\mu\right]\right\}$, there exists $t_{4}>t_{3}$, such that $P_{k}(t) \geq x_{k}^{(1)}+\tau_{4}$ for $t>t_{4}$, where

$$
x_{k}^{(1)}=\frac{\mu}{\alpha k\left(\rho_{1}+\rho_{2}\right) \tau+\mu}-2 \tau_{4}>0 .
$$

Then, substituting $\theta_{1}(t)>\tau, \theta_{2}(t)>\tau$ into system (8), it follows that

$\frac{\mathrm{d} M_{k}(t)}{\mathrm{d} t} \geq k \alpha\left(\rho_{1}+\rho_{2}\right) \tau x_{k}^{(1)}-\left(w_{1}+\mu+\varphi\right) M_{k}(t)+f \tau, \quad t>t_{4}$.

Hence, for constant $0<\tau_{5}<\min \{1 / 5, k \alpha$ $\left.\left(\rho_{1}+\rho_{2}\right) \tau x_{k}^{(1)}+f \tau / 2\left(w_{1}+\mu+\varphi\right)\right\}$, there exists $t_{5}>t_{4}$, such that $M_{k}(t) \geq y_{k}^{(1)}+\tau_{5}$ for $t>t_{5}$, where

$$
y_{k}^{(1)}=\frac{k \alpha\left(\rho_{1}+\rho_{2}\right) \tau x_{k}^{(1)}+f \tau}{w_{1}+\mu+\varphi}-2 \tau_{5}>0 .
$$

Therefore,

$$
\frac{\mathrm{d} S_{k}(t)}{\mathrm{d} t} \geq \varphi y_{k}^{(1)}-\left(w_{2}+\mu\right) S_{k}(t), \quad t>t_{5} .
$$

Hence, for constant $0<\tau_{6}<\min \left\{1 / 6, \tau_{5}\right.$, $\left.\varphi y_{k}^{(1)} / 2\left(w_{2}+\mu\right)\right\}$, there exists $t_{6}>t_{5}$, such that $S_{k}(t) \geq z_{k}^{(1)}+$ $\tau_{6}$ for $t>t_{6}$, where

$$
z_{k}^{(1)}=\frac{\varphi y_{k}^{(1)}}{w_{2}+\mu}-2 \tau_{6}>0 .
$$

Because $\tau$ is a small positive constant, we can get

$$
\begin{gathered}
0<x_{k}^{(1)}<P_{k}<X_{k}^{(1)}<1, \\
0<y_{k}^{(1)}<M_{k}<Y_{k}^{(1)}<1, \\
0<z_{k}^{(1)}<S_{k}<Z_{k}^{(1)}<1 .
\end{gathered}
$$

Let

$$
\begin{aligned}
q^{(j)} & =\sum_{i=1}^{n} P_{i}\left(y_{i}^{(j)}+z_{i}^{(j)}\right), \\
Q^{(j)} & =\sum_{i=1}^{n} P_{i}\left(Y_{i}^{(j)}+Z_{i}^{(j)}\right), \\
j & =1,2, \ldots, n .
\end{aligned}
$$


From the above discussion, we have

$$
0<q^{(1)} \leq\left(\rho_{1} \theta_{1}+\rho_{2} \theta_{2}\right) \leq Q^{(1)}<\rho_{1}+\rho_{2}, \quad t>t_{6} .
$$

And, we substitute $M_{k}(t) \geq y_{k}^{(1)}+\tau_{5}, S_{k}(t) \geq z_{k}^{(1)}+\tau_{6}$ into system (8), we can get

$$
\frac{\mathrm{d} P_{k}(t)}{\mathrm{d} t} \leq \mu-\left(k \alpha q^{(1)}+\mu\right) P_{k}(t), \quad t>t_{6} .
$$

Consequently, for constant $0<\tau_{7}<\min \left\{1 / 7, \tau_{6}\right\}$, there exists $t_{7}>t_{6}$, such that

$$
P_{k}(t) \leq X_{k}^{(2)} \triangleq \min \left\{X_{k}^{(1)}-\tau_{1}, \frac{\mu}{\mu+k \alpha q^{(1)}}+\tau_{7}\right\}, \quad t>t_{7} .
$$

Thus,

$$
\begin{aligned}
\frac{\mathrm{d} M_{k}(t)}{\mathrm{d} t} \leq & k \alpha Q^{(1)} X_{k}^{(2)}-\left(w_{1}+\varphi+\mu\right) M_{k}(t) \\
& +f\left(1-M_{k}(t)-x_{k}^{(1)}-z_{k}^{(1)}\right) \\
\leq & k \alpha Q^{(1)} X_{k}^{(2)}-\left(w_{1}+\varphi+\mu\right) M_{k}(t) \\
& +f\left(1-x_{k}^{(1)}-z_{k}^{(1)}\right), \quad t>t_{7} .
\end{aligned}
$$

Consequently, for constant $0<\tau_{8}<\min \left\{1 / 8, \tau_{7}\right\}$, there exists $t_{8}>t_{7}$, such that

$$
\begin{aligned}
M_{k}(t) \leq Y_{k}^{(2)} \triangleq & \min \left\{Y_{k}^{(1)}\right. \\
& \left.-\tau_{2}, \frac{k Q^{(1)} X_{k}^{(2)}+f\left(1-x_{k}^{(1)}-z_{k}^{(1)}\right)}{w_{1}+\varphi+\mu+f}+\tau_{8}\right\}, \quad t>t_{8} .
\end{aligned}
$$

As a result, it follows that

$$
\frac{\mathrm{d} S_{k}(t)}{\mathrm{d} t} \leq \varphi M_{k}^{(2)}-\left(w_{2}+\mu\right) S_{k}(t), \quad t>t_{9} .
$$

Therefore, for constant $0<\tau_{9}<\min \left\{1 / 9, t \tau_{8}\right\}$, we can get

$$
S_{k}(t) \leq Z_{k}^{(2)} \triangleq \min \left\{Z_{k}^{(1)}-\tau_{3}, \frac{\varphi Y_{k}^{(2)}}{w_{2}+\mu}+\tau_{9}\right\}, \quad t>t_{9} .
$$

Substituting $\quad M_{k}(t) \leq Y_{k}^{(2)}, S_{k}(t) \leq Z_{k}^{(2)} \quad$ and $\quad \rho_{1} \theta_{1}+$ $\rho_{2} \theta_{2} \leq Q^{(2)}$ into system (8), where

$$
\frac{\mathrm{d} P_{k}(t)}{\mathrm{d} t} \geq \mu-\left(k \alpha Q^{(2)}+\mu\right) P_{k}(t), \quad t>t_{9} .
$$

Hence, for constant $0<\tau_{10}<\min \{1 / 10, t$ $\left.\tau_{9} n, q \mu / 2\left(k \alpha Q^{(2)}+\mu\right)\right\}$, there exists $t_{10}>t_{9}$, such that $P_{k}(t) \geq x_{k}^{(2)}+\tau_{10}$ for $t>t_{10}$, where

$$
x_{k}^{(2)}=\max \left\{x_{k}^{(1)}+\tau_{4}, \frac{\mu}{\mu+k \alpha Q^{(2)}}-2 \tau_{10}\right\} .
$$

Thus,

$$
\begin{aligned}
\frac{\mathrm{d} M_{k}(t)}{\mathrm{d} t} \geq & k \alpha q^{(1)} x_{k}^{(2)}-\left(\varphi+w_{1}+\mu\right) M_{k}(t) \\
& +f\left(1-M_{k}(t)-x_{k}^{(1)}-z_{k}^{(1)}\right), \quad t>t_{10} .
\end{aligned}
$$

So, for constant $0<\tau_{11}<\min \left\{1 / 11, t \tau_{10} n, q k \alpha q^{(1)}\right.$ $\left.x_{k}^{(2)}+f\left(1-x_{k}^{(1)}-z_{k}^{(1)}\right) / 2\left(w_{1}+\varphi+\mu+f\right)\right\}$, there exists $t_{11}>t_{10}$, and $M_{k}(t) \geq y_{k}^{(2)}+\tau_{11}$ for $t>t_{11}$, where

$$
y_{k}^{(2)}=\max \left\{y_{k}^{(1)}+\tau_{5}, \frac{k \alpha q^{(1)} x_{k}^{(2)}+f\left(1-x_{k}^{(1)}-z_{k}^{(1)}\right)}{w+f+\varphi+\mu}-2 \tau_{11}\right\} .
$$

Similarly,

$$
\frac{\mathrm{d} S_{k}(t)}{\mathrm{d} t} \geq \varphi y_{k}^{(2)}-\left(w_{2}+\mu\right) S_{k}(t), \quad t>t_{11} .
$$

Therefore, for constant $0<\tau_{12}<\min \left\{1 / 12, t \tau_{11} n, q\right.$ $\left.\varphi y_{k}^{(2)} / 2\left(w_{2}+\mu\right)\right\}$, there exists $t_{12}>t_{11}$, and $S_{k}(t) \geq z_{k}^{(2)}+\tau_{12}$ for $t>t_{12}$, where

$$
z_{k}^{(2)}=\max \left\{z_{k}^{(1)}+\tau_{6}, \frac{\varphi y_{k}^{(2)}}{w_{2}+\mu}-2 \tau_{12}\right\} .
$$

According to the above discussion and analyses, we can obtain six sequences: $\left\{X_{k}^{(l)}\right\},\left\{Y_{k}^{(l)}\right\},\left\{Z_{k}^{(l)}\right\},\left\{x_{k}^{(l)}\right\},\left\{y_{k}^{(l)}\right\}$, and $\left\{z_{k}^{(l)}\right\}$. We can find that the first three sequences are monotonically increasing, and the last three sequences are strictly monotonically decreasing, and there is a sufficiently large positive integer $H$ such that, for $l \geq H$,

$$
\left\{\begin{array}{l}
X_{k}^{(l)}=\frac{\mu}{\mu+k \alpha q^{(l-1)}}+\tau_{6 l-5}, \\
Y_{k}^{(l)}=\frac{k \alpha Q^{(l-1)} X_{k}^{(l)}+f\left(1-x_{k}^{(l-1)}-z_{k}^{(l-1)}\right)}{w_{1}+\varphi+f+\mu}+\tau_{6 l-4}, \\
Z_{k}^{(l)}=\frac{\varphi Y_{k}^{(l)}}{w_{2}+\mu}+\tau_{6 l-3}, \\
x_{k}^{(l)}=\frac{\mu}{\mu+k \alpha Q^{(l)}}-2 \tau_{6 l-2}, \\
y_{k}^{(l)}=\frac{k \alpha q^{(l-1)} x_{k}^{(l)}+f\left(1-X_{k}^{(h)}-Z_{k}^{(h)}\right)}{w_{1}+\varphi+f+\mu}-2 \tau_{6 l-1}, \\
z_{k}^{(l)}=\frac{\varphi y_{k}^{(l)}}{w_{2}+\mu}-2 \tau_{6 l-1} .
\end{array}\right.
$$

It is easy to find that 


$$
\begin{aligned}
x_{k}^{(l)} & \leq P_{k}(t) \leq X_{k}^{(l)}, \\
y_{k}^{(l)} & \leq M_{k}(t) \leq Y_{k}^{(l)}, \\
z_{k}^{(l)} & \leq S_{k}(t) \leq Z_{k}^{(l)}, \\
t & >t_{6 l} .
\end{aligned}
$$

Since the sequential limits of system (60) exist, let $\lim _{l \longrightarrow \infty} \Omega_{k}^{(l)}=\Omega_{k}, \quad$ where $\quad \Omega_{k}^{(l)} \in\left\{X_{k}^{(l)}, Y_{k}^{(l)}, Z_{k}^{(l)}\right.$, $\left.x_{k}^{(l)}, y_{k}^{(l)}, z_{k}^{(l)}, Q_{k}^{(l)}, q_{k}^{(l)}\right\} \quad$ and $\quad \Omega_{k} \in\left\{X_{k}, Y_{k}, Z_{k}, x_{k}, y_{k}, z_{k}\right.$, $\left.Q_{k}, q_{k}\right\}$.

Since $0<\tau_{l}<1 / l$, it has $\tau_{l} \longrightarrow 0$ as $l \longrightarrow \infty$. Supposing $l \longrightarrow \infty$, it follows from (61) that

$$
\begin{aligned}
& X_{k}=\frac{\mu}{k \alpha q+\mu}, \\
& Y_{k}=\frac{k \alpha Q X_{k}+f\left(1-x_{k}-z_{k}\right)}{w_{1}+f+\mu+\varphi}, \\
& Z_{k}=\frac{\varphi}{w_{2}+\mu} Y_{k}, \\
& x_{k}=\frac{\mu}{k \alpha Q+\mu}, \\
& y_{k}=\frac{k \alpha q x_{k}+f\left(1-X_{k}-Z_{k}\right)}{w_{1}+f+\mu+\varphi}, \\
& z_{k}=\frac{\varphi}{w_{2}+\mu} y_{k},
\end{aligned}
$$

where

$$
\begin{gathered}
q=\sum_{i=1}^{n} P_{i}\left(y_{i}+z_{i}\right), \\
Q=\sum_{i=1}^{n} P_{i}\left(Y_{i}+Z_{i}\right) .
\end{gathered}
$$

Furthermore,

$$
\begin{aligned}
Y_{k} & =\frac{\left\{k Q \alpha\left[\left(w_{2}+\mu\right)\left(w_{2}+\mu\right)(k \alpha Q \mu+f(k \alpha q+\mu))+\varphi f(k \alpha q+\mu)-(k \alpha q+\mu)\left(f\left(w_{2}+\mu\right)\left(w_{2}+\mu\right)+\varphi k \alpha q\right)+f \varphi\right]\right\}}{\left\{(k \alpha q+\mu)(k \alpha Q+\mu)\left(w_{1}+f+\mu+\varphi\right)\left(w_{2}+\mu\right)\left(w_{2}+\mu\right)\right\}}, \\
y_{k} & =\frac{k q \alpha\left[\left\{\left(w_{2}+\mu\right)\left(w_{2}+\mu\right)(k \alpha q \mu+f(k \alpha Q+\mu))+\varphi f(k \alpha Q+\mu)\right\}-(k \alpha Q+\mu)\left(f\left(w_{2}+\mu\right)\left(w_{2}+\mu\right)+\varphi k \alpha Q\right)+f \varphi\right]}{(k \alpha q+\mu)(k \alpha Q+\mu)\left(w_{1}+f+\mu+\varphi\right)\left(w_{2}+\mu\right)\left(w_{2}+\mu\right)} .
\end{aligned}
$$

Substituting (62) and (64) into $Q$ and $q$, respectively, we obtain

$$
\begin{aligned}
& 1=\frac{\varphi+w_{2}+\mu}{w_{2}+\mu} \sum_{i=1}^{n} i P_{i}\left\{\frac{\left\{k \alpha\left[\left(w_{2}+\mu\right)\left(w_{2}+\mu\right)(k \alpha Q \mu+f(k \alpha q+\mu))+\varphi f(k \alpha q+\mu)\right]-(k \alpha q+\mu)\left[\left(f\left(w_{2}+\mu\right)\left(w_{2}+\mu\right)+\varphi k \alpha q\right)+f \varphi\right]\right\}}{(k \alpha q+\mu)(k \alpha Q+\mu)\left(w_{1}+f+\mu+\varphi\right)\left(w_{2}+\mu\right)\left(w_{2}+\mu\right)}\right\}, \\
& 1=\frac{\varphi+w_{2}+\mu}{w_{2}+\mu} \sum_{i=1}^{n} i P_{i}\left\{\frac{\left\{k \alpha\left[\left(w_{2}+\mu\right)\left(w_{2}+\mu\right)(k \alpha q \mu+f(k \alpha Q+\mu))+\varphi f(k \alpha Q+\mu)\right]-(k \alpha Q+\mu)\left[\left(f\left(w_{2}+\mu\right)\left(w_{2}+\mu\right)+\varphi k \alpha Q\right)+f \varphi\right]\right\}}{(k \alpha q+\mu)(k \alpha Q+\mu)\left(w_{1}+f+\mu+\varphi\right)\left(w_{2}+\mu\right)\left(w_{2}+\mu\right)}\right\}
\end{aligned}
$$




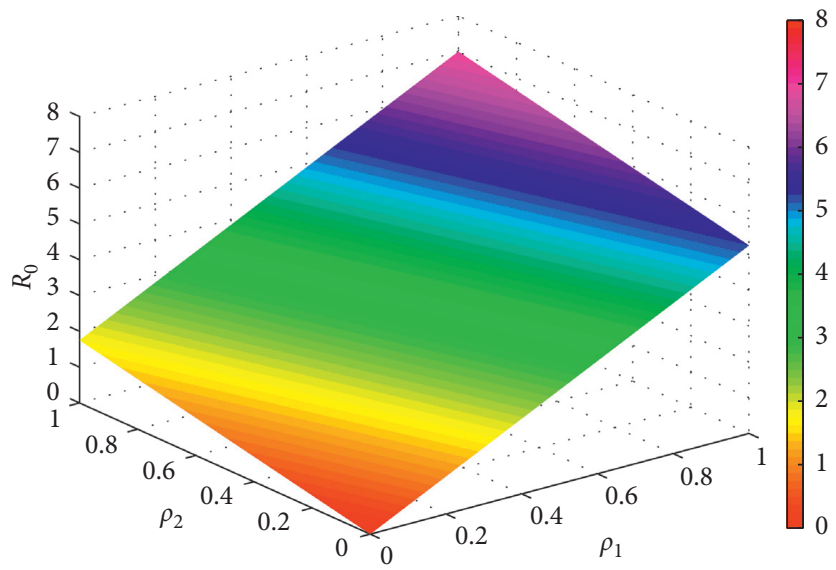

FIgURE 2: The influence of parameters $\rho_{1}$ and $\rho_{2}$ on $R_{0}$.

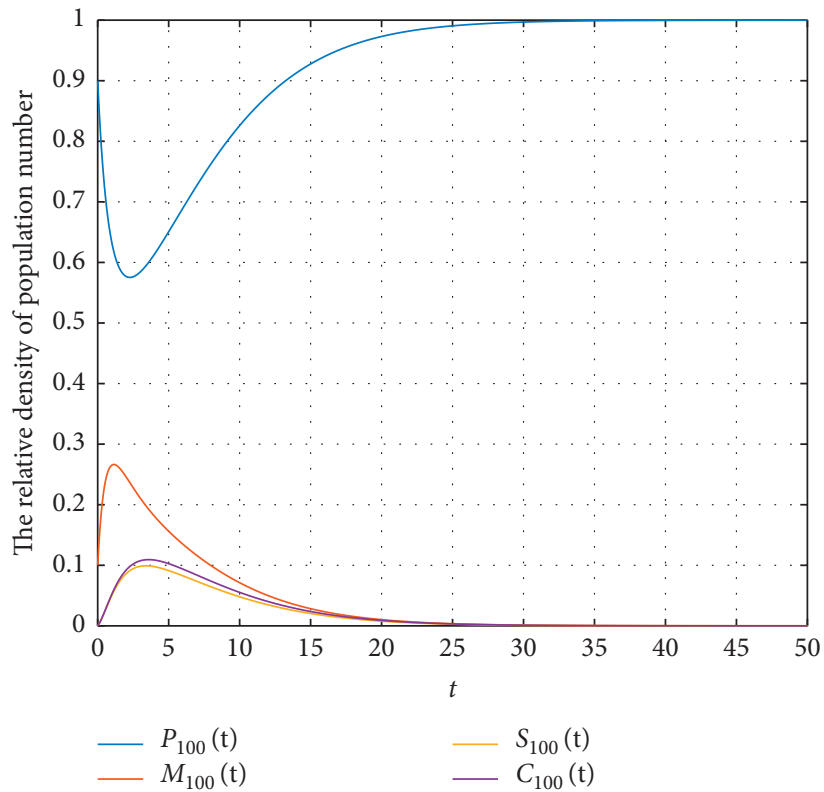

Figure 3: The time series and orbits of four states with $R_{0}<1$.

From the above two equations, we can get

$$
(Q-q) \frac{\varphi+w_{2}+\mu}{w_{2}+\mu} \sum_{i=1}^{n} i P_{i}\left\{\frac{[(k a q+\mu)-(k a Q+\mu)]\left[\left(f\left(w_{2}+\mu\right)\left(w_{2}+\mu\right)+\varphi k a Q\right)+f \varphi\right]}{(k a q+\mu)(k a Q+\mu)\left(w_{1}+f+\mu+\varphi\right)\left(w_{2}+\mu\right)\left(w_{2}+\mu\right)}\right\} \equiv 0 .
$$

It is clear that $Q=q$. So, $\sum_{i=1}^{n} P_{i}\left[\left(Y_{i}-y_{i}\right)+\right.$ $\left.\left(Z_{i}-z_{i}\right)\right]=0$, which is equivalent to $Y_{i}=y_{i}$ and $Z_{i}=z_{i}$ for $1 \leq i \leq n$. Then, from systems (61) and (62), it can be concluded that

$$
\begin{aligned}
& \lim _{t \longrightarrow \infty} P_{k}(t)=X_{k}=x_{k}, \\
& \lim _{t \longrightarrow \infty} M_{k}(t)=Y_{k}=y_{k}, \\
& \lim _{t \longrightarrow \infty} R_{k}(t)=Z_{k}=z_{k} .
\end{aligned}
$$




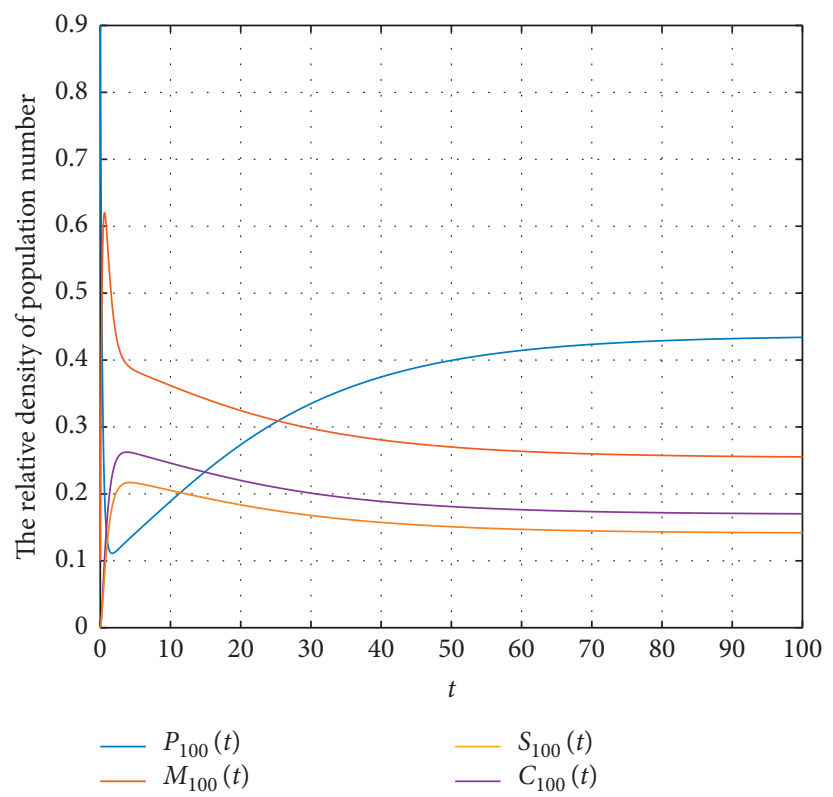

Figure 4: The time series and orbits of four states with $R_{0}>1$.

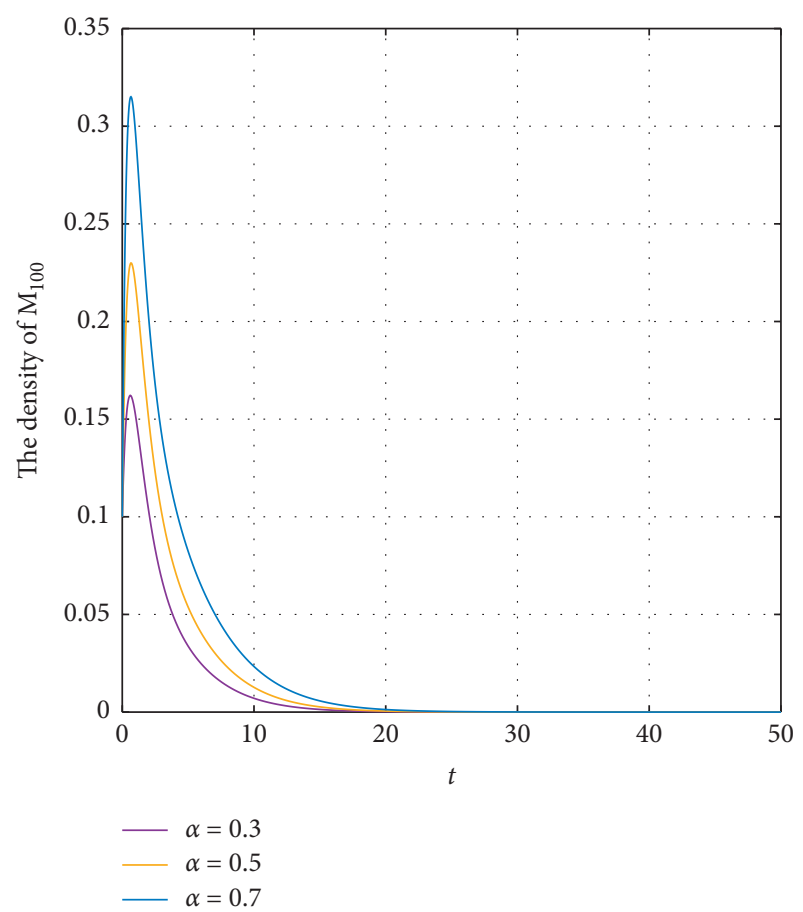

(a)

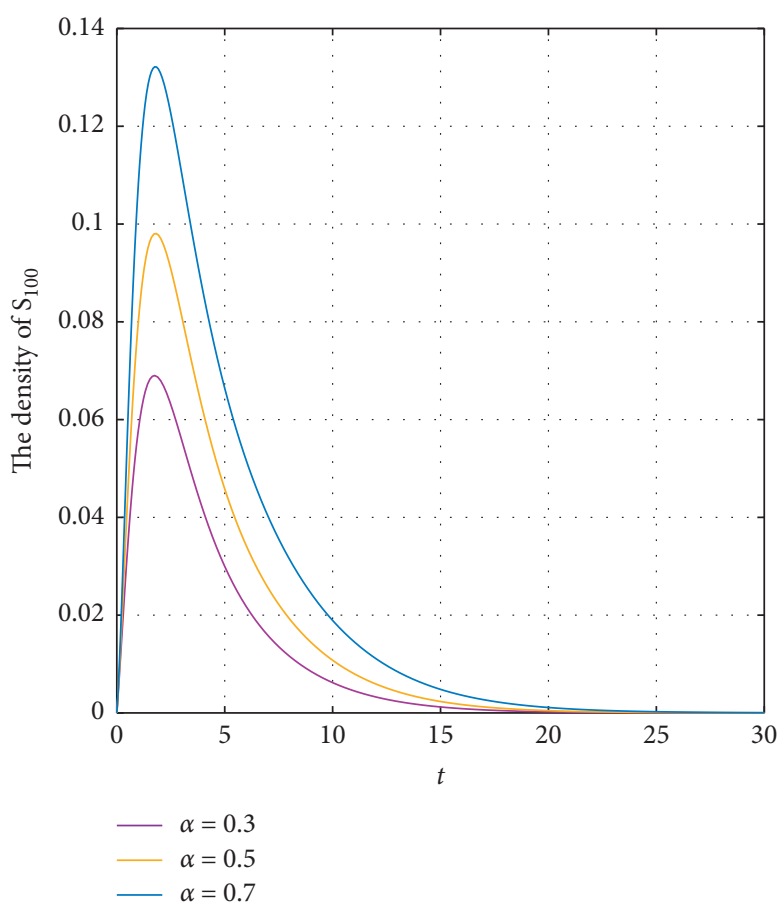

(b)

Figure 5: The prevalence of $M_{100}$ and $S_{100}$ versus $t$ to different $\alpha$ with $R_{0}<1$.

Finally, $Q=q$ is substituted into system (64). For system (62), we can get $X_{k}=P_{k}^{*}, Y_{k}=M_{k}^{*}$, and $Z_{k}=R_{k}^{*}$. This proof is completed.

\section{Numerical Simulations}

This section illustrates the analytical results through numerical simulations. Based on scale-free network, we have
$H(k)=c k^{-3}$ in system (1), and the parameter $c$ satisfies $\sum_{k=1}^{n} c k^{-3}=1, n=1000$.

In Figure 2, the parameters are chosen as $\mu=0.3$, $\alpha=0.2, \varphi=0.3, w_{2}=0.5, w_{1}=0.3$, and $f=0.6$. We can see that the larger the $\rho_{1}$ and $\rho_{2}$, the larger the $R_{0}$, i.e., long time contact with mild addicts and severe addicts both can increase the spreading speed of betel nut addiction. This also means that if people's health knowledge is improved, the 


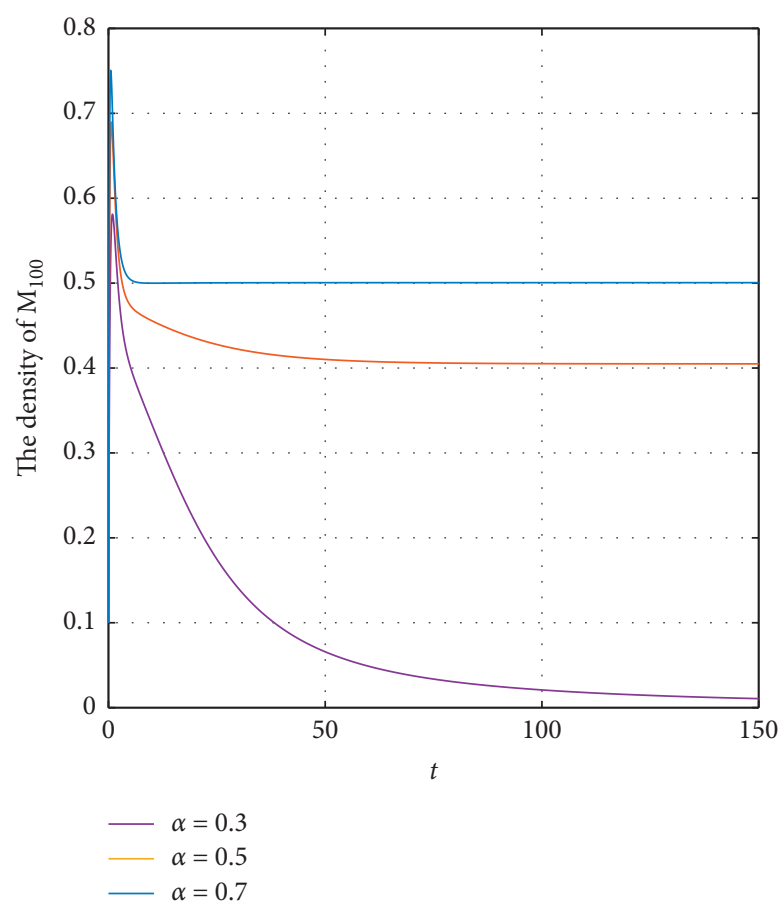

(a)

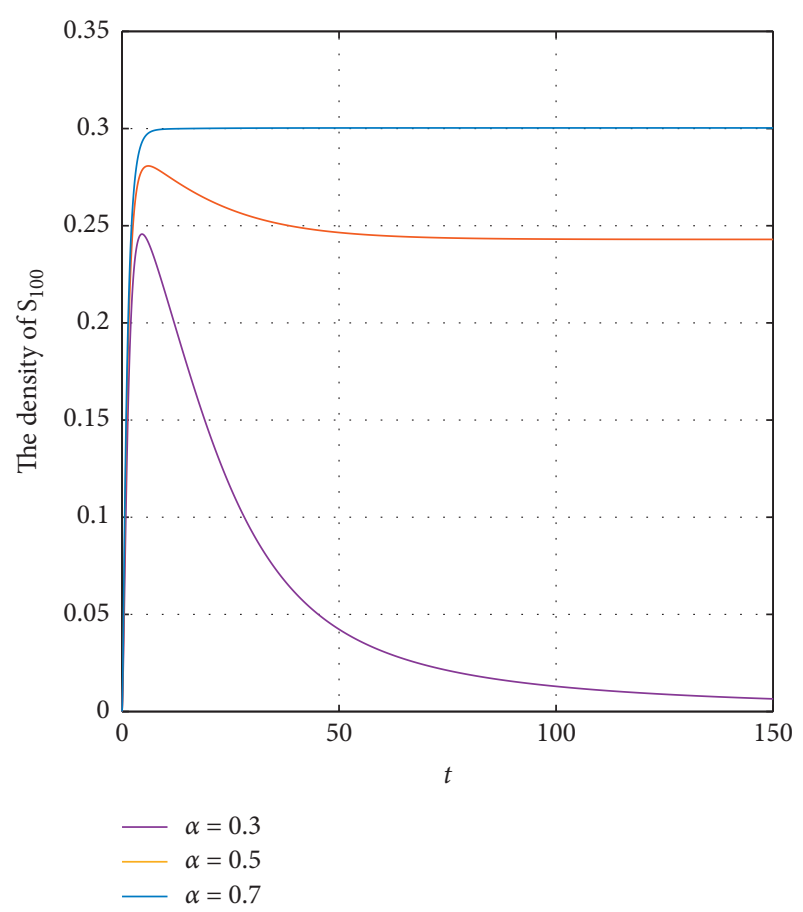

(b)

Figure 6: The prevalence of $M_{100}$ and $S_{100}$ versus $t$ to different $\alpha$ with $R_{0}>1$.

probability of people chewing betel nut is reduced. The spreading of betel nut addiction will be weakened.

In Figure 3, we choose $\mu=0.3, \alpha=0.2, \varphi=0.4, w_{2}=0.4$, $w_{1}=0.3, f=0.6, \rho_{1}=0.4$, and $\rho_{2}=0.4$, thus the basic reproductive number $R_{0}=0.8090<1$. We can see that there is almost no spreading of betel nut addiction when $R_{0}<1$, which means that betel nut addiction will eventually disappear. It also suggests that the addiction-elimination equilibrium $E_{0}$ is globally asymptotically stable when $R_{0}<1$. This also means that restricting the marketing strategy of betel nut and controlling the recurrence rate of betel nut addiction will weaken the spreading of betel nut addiction.

In Figure 4, we choose $\mu=0.3, \alpha=0.5, \varphi=0.4, w_{2}=0.3$, $w_{1}=0.3, f=0.6, \rho_{1}=0.7$, and $\rho_{2}=0.7$, thus the basic reproductive number $R_{0}=3.7163>1$. We can see that the number of mild addicts and severe addicts will maintain at a positive constant, i.e., the spreading phenomenon of betel nut addiction is permanent on the scale-free networks when $R_{0}>1$.

In Figure 5, we choose $\mu=0.4, \varphi=0.6, w_{2}=0.6$, $w_{1}=0.4, f=0.2, \rho_{1}=0.2$, and $\rho_{2}=0.2$, thus the basic reproductive number $R_{0}<1$. We can see that the corresponding $M_{100}$ and $S_{100}$ decrease significantly as the parameter $\alpha$ decreases, i.e., a smaller parameter $\alpha$ can weaken the spreading phenomenon of betel nut addiction. This also means that the marketing strategies of betel nut merchants have a great influence on the spreading of betel nut addiction.

In Figure 6, we choose $\mu=0.3, \varphi=0.4, w_{2}=0.3$, $w_{1}=0.3, f=0.6, \rho_{1}=0.7$, and $\rho_{2}=0.7$, thus the basic reproductive number $R_{0}>1$. Figure 6 describes the change of the mild addicts $M_{100}$ and severe addicts $S_{100}$ with different rate $\alpha$. We can see that when $R_{0}>1, M_{100}$ and $S_{100}$ both grow to positive constants with the increasing of parameter $\alpha$. This figure shows that a larger $\alpha$ can lead to the larger value of betel nut addiction spreading level. It means that restricting the marketing strategies of betel nut plays a vital role in controlling the spread of betel nut addiction.

\section{Conclusions}

In this paper, a new comprehensively PMSR betel nut addiction spreading model has been proposed. By the mean-field theory, we have obtained the basic reproduction number $R_{0}$ addiction-elimination equilibrium $E_{0}$, and addiction-prevailing equilibrium $E_{+}$If $R_{0}<1$, the addiction-elimination equilibrium $E_{0}$ is globally asymptotically stable, i.e., betel nut addiction spreading behavior will eventually disappear. If $R_{0}>1$, the spreading dynamical behavior of betel nut addiction is persistent and globally asymptotically stable. Moreover, we found that the larger the parameters $\rho_{1}$ and $\rho_{2}$, the larger the basic reproductive number $R_{0}$, i.e., long-term and frequent contact with mild addicts and severe addicts can increase the spreading phenomenon of betel nut addiction. Furthermore, a larger $\alpha$ can increase the spread speed and range of betel nut addiction. Therefore, controlling the spread of betel nut addiction requires restricting the marketing strategies adopted by betel nut merchants and widely publicizing the harmfulness of chewing betel nut. The research results have important guiding significance in controlling the spreading of betel nut addiction. 


\section{Data Availability}

The data used to support the findings of this study are available from the corresponding author upon request.

\section{Conflicts of Interest}

The authors declare that there are no conflicts of interest regarding the publication of this paper.

\section{Acknowledgments}

This work was supported in part by the National Natural Science Foundation of China under grant nos. 61672112 and 61873287.

\section{References}

[1] Y.-C. Ko, Y.-L. Huang, C.-H. Lee, M.-J. Chen, L.-M. Lin, and C.-C. Tsai, "Betel quid chewing, cigarette smoking and alcohol consumption related to oral cancer in Taiwan," Journal of Oral Pathology and Medicine, vol. 24, no. 10, pp. 450-453, 1995.

[2] C.-H. Lee, Y.-C. Ko, H.-L. Huang et al., "The precancer risk of betel quid chewing, tobacco use and alcohol consumption in oral leukoplakia and oral submucous fibrosis in southern Taiwan," British Journal of Cancer, vol. 88, no. 3, p. 366, 2003.

[3] A. Znaor, P. Brennan, V. Gajalakshmi et al., "Independent and combined effects of tobacco smoking, chewing and alcohol drinking on the risk of oral, pharyngeal and esophageal cancers in Indian men," International Journal of Cancer, vol. 105, no. 5, pp. 681-686, 2003.

[4] P. C. Gupta and S. Warnakulasuriya, "Global epidemiology of areca nut usage," Addiction Biology, vol. 7, no. 1, pp. 77-83, 2002.

[5] B. J. Boucher and N. Mannan, "Metabolic effects of the consumption of areca catechu," Addiction Biology, vol. 7, no. 1, pp. 103-110, 2002.

[6] S. Warnakulasuriya, C. Trivedy, and T. J. Peters, "Areca nut use: an independent risk factor for oral cancer," The Health Problem is Under-Recognised, vol. 324, no. 799, 2002.

[7] H. Mehrtash, K. Duncan, M. Parascandola et al., "Defining a global research and policy agenda for betel quid and areca nut," Lancet Oncology, vol. 18, no. 12, 2017.

[8] C. Trivedy, S. Warnakulasuriya, and T. J. Peters, "Areca nuts can have deleterious effects," BMJ, vol. 318, no. 7193, p. 1287, 1999.

[9] M. A. Lopezvilchez, V. Seidel, M. Farre et al., "Areca-nut abuse and neonatal withdrawal syndrome," Pediatrics, vol. 117 , no. $1,2006$.

[10] J.-C. Weng, T.-W. Kao, G.-J. Huang, Y.-S. Tyan, H.-C. Tseng, and M.-C. Ho, "Evaluation of structural connectivity changes in betel-quid chewers using generalized q-sampling MRI," Psychopharmacology, vol. 234, no. 13, pp. 1945-1955, 2017.

[11] Science Daily, "Researcher finds key clues about "betel nut" addiction that plagues millions worldwide," October 2015, https:// www.sciencedaily.com/releases/2015/10/151021161034.htm.

[12] K. L. Murphy and T. A. Herzog, "Sociocultural factors that affect chewing behaviors among betel nut chewers and exchewers on guam," Hawai'i Journal of Medicine \& Public Health, vol. 74, no. 12, pp. 406-411, 2015.

[13] C.-H. Lee, A. M.-S. Ko, S. Warnakulasuriya et al., "Intercountry prevalences and practices of betel-quid use in south, southeast and eastern asia regions and associated oral preneoplastic disorders: an international collaborative study by asian betel-quid consortium of south and east Asia," International Journal of Cancer, vol. 129, no. 7, pp. 1741-1751, 2011.

[14] W. M. Ghani, I. A. Razak, Y. Yang et al., "Factors affecting commencement and cessation of betel quid chewing behaviour in Malaysian adults," BMC Public Health, vol. 11, no. 1, p. 82, 2011.

[15] J. Moss, C. Kawamoto, P. Pokhrel et al., "Developing a betel quid cessation program on the island of guam," Pacific Asia inquiry, vol. 6, no. 1, pp. 144-150, 2015.

[16] A. Ariyawardana, A. Athukorala, and A. Arulanandam, "Effect of betel chewing, tobacco smoking and alcohol consumption on oral submucous fibrosis: a case-control study in Sri Lanka," Journal of Oral Pathology and Medicine, vol. 35, no. 4, pp. 197-201, 2006.

[17] C.-C. Lin, I. Tamí-Maury, W.-F. Ma et al., "Social and cultural context of betel quid consumption in taiwan and implications for prevention and cessation interventions," Substance Use \& Misuse, vol. 52, no. 5, pp. 646-655, 2017.

[18] H. A. Seedat and C. W. van Wyk, "Betel chewing and dietary habits of chewers without and with submucous fibrosis and with concomitant oral cancer," South African Medical Journal = Suid-Afrikaanse Tydskrif Vir Geneeskunde, vol. 74, no. 11, p. 572, 1988.

[19] S. Williams, A. Malik, S. Chowdhury, and S. Chauhan, "Sociocultural aspects of areca nut use," Addiction Biology, vol. 7, no. 1, pp. 147-154, 2002.

[20] X. Liu, T. Li, H. Xu, and W. Liu, "Spreading dynamics of an online social information model on scale-free networks," Physica A: Statistical Mechanics and Its Applications, vol. 514, pp. 497-510, 2019.

[21] Y. Lei, T. Li, Y. Wang, G. Ye, S. Sun, and Z. Xia, "Spreading dynamics of a CPFB group booking preferential information model on scale-free networks," IEEE Access, vol. 7, pp. 156287-156300, 2019.

[22] X. Liu, T. Li, M. Tian et al., "Rumor spreading of a SEIR model in complex social networks with hesitating mechanism," Advances in Difference Equations, vol. 2018, no. 1, pp. 1-24, Article ID 391, 2018.

[23] T. Li, Y. Wang, Z.-H. Guan et al., "Spreading dynamics of a SIQRS epidemic model on scale-free networks," Communications in Nonlinear Science and Numerical Simulation, vol. 19, no. 3, pp. 686-692, 2014.

[24] Y. Wang, Z. Jin, Z. Yang, Z.-K. Zhang, T. Zhou, and G.-Q. Sun, "Global analysis of an SIS model with an infective vector on complex networks," Nonlinear Analysis: Real World Applications, vol. 13, no. 2, pp. 543-557, 2012.

[25] Y. Wang and J. Cao, "Global dynamics of a network epidemic model for waterborne diseases spread," Applied Mathematics and Computation, vol. 237, pp. 474-488, 2014.

[26] W. Liu, T. Li, X. Cheng et al., "Spreading dynamics of a cyber violence model on scale-free networks," Physica A: statistical mechanics and its applications, vol. 531, Article ID 121752, 2019.

[27] A.-L. Barabási and R. Albert, "Emergence of scaling in random networks," Science, vol. 286, no. 5439, pp. 509-512, 1999.

[28] R. Albert and A. Barabasi, "Statistical mechanics of complex networks," Reviews of Modern Physics, vol. 74, no. 1, pp. 47-97, 2001.

[29] M. Liu, G. Sun, Z. Jin, and T. Zhou, "An analysis of transmission dynamics of drug-resistant disease on scale-free networks," Applied Mathematics and Computation, vol. 222, pp. 177-189, 2013. 
[30] R. Pastor-Satorras and A. Vespignani, "Epidemic spreading in scale-free networks," Physical Review Letters, vol. 86, no. 14, p. $3200,2001$.

[31] J. C. Nacher, M. Hayashida, and T. Akutsu, "Emergence of scale-free distribution in protein-protein interaction networks based on random selection of interacting domain pairs," BioSystems, vol. 95, no. 2, pp. 155-159, 2009.

[32] P. Van den Driessche and J. Watmough, "Reproduction numbers and sub-threshold endemic equilibria for compartmental models of disease transmission," Mathematical Biosciences, vol. 180, no. 1-2, pp. 29-48, 2002.

[33] A. Lajmanovich and J. A. Yorke, "A deterministic model for gonorrhea in a nonhomogeneous population," Mathematical Biosciences, vol. 28, no. 3-4, pp. 221-236, 1976.

[34] F. Chen, "On a nonlinear nonautonomous predator-prey model with diffusion and distributed delay," Journal of Computational and Applied Mathematics, vol. 180, no. 1, pp. 33-49, 2005.

[35] G. Zhu, X. Fu, and G. Chen, "Spreading dynamics and global stability of a generalized epidemic model on complex heterogeneous networks," Applied Mathematical Modelling, vol. 36, no. 12, pp. 5808-5817, 2012. 